\title{
System Organization of Mental Activity of the Brain during Wakefulness and Sleep
}

\author{
*Yumatov Evgeny \\ P. K. Anokhin Research Institute of Normal Physiology, Russia
}

Submission: February 15, 2017; Published: February 28, 2017

*Corresponding author: Yumatov Evgeny, IM Sechenov First Moscow State Medical University, P. K. Anokhin Research Institute of Normal Physiology, Moscow, Russia, Email: eayumatov@mail.ru

\begin{abstract}
The article deals with the problem of mental activity of the brain during wakefulness and sleep. It is noted that all the current understanding of sleep and wakefulness are based only on the study of neurophysiological processes, without taking into account the mechanisms of mental activity brain that are still poorly understood. It is shown that the mental state of a person, and sleep are closely related. Propose new approaches to the study of the nature of the mental activity of the brain, based on the methodological principle of "mental processes can directly register and study only with the help and participation of living structures." The basic scheme of the systemic organization of mental activity of the brain during sleep and while awake.
\end{abstract}

Keywords: Psyche; Mind; Brain; Sleep

\section{Introduction}

The brain is a unique organization in nature, having the mental activity, which is expressed in thoughts, feelings, emotions, ie, in the subjective perception of the man himself and the world. Knowledge of the nature of mental activity of the brain is the most urgent and the most challenging task of physiology [1-11]. The mental activity of the brain appears during sleep and wakefulness. This mental activity is associated only with wakefulness, and while it remains outside the field of view of researchers. Sleep and wakefulness - two interconnected natural physiological state, which is periodically constantly living organism. It can be argued that without taking into account the mental activity of the brain can not understand the nature of sleep and wakefulness.

Wakefulness is directly related to the mental activity of the brain. Presence awareness allows all forms of active interaction of organism and environment: sensory perception, memory, learning, behavior, and so forth. During waking hours the body carries out satisfaction of vital social and biological needs: nutritious, protective and territorial, cognitive, creative, social, gender and so on.

Sleep - the physiological state of the higher animals and humans, is characterized by the temporary shutdown of consciousness, lack of active targeted behavioral activity and a general decline in metabolism. However, during sleep keep your mental activity of the brain in the form of emotional states, feelings, dreams, and so forth.

Sleep is a vital necessity, determines the mental state of man, his mood, feeling, memory performance. Sleep disorders are often the first symptom of mental illness. Sleep is essential for normal mental activity of the brain during wakefulness. Knowledge of the nature of mental activity of the brain is one of the most challenging problems of science. This also applies to the study of mental activity of the brain during sleep. To know the nature of sleep - it means understand the biological role of sleep and cause neurophysiological and mental activity of the brain during sleep; the origin and the need for sleep phase structure, mechanisms of natural awakening after enough sleep. These criteria shall comply with the theory of sleep.

\section{Theories of sleep}

On the basis of individual experimental studies and observations were formulated various theories of sleep.

I. Chemical dream theory - explains the development of sleep accumulation in the body of specific substances. The 
basis for this theory served as an experiment, in which the waking dog transfused blood of the animal, deprived of sleep for days. After that, the animal recipient immediately fell asleep. One of the identified endogenous substance which stimulates sleep, sleep delta peptide is [12]. However, there is still no information on the specific substances that are formed during wakefulness and are factors that shape sleep motivation. Observations of the behavior of Siamese twins with a common circulatory system, show that. These twins could sleep at different times, one of them could sleep, while the other watched.

II. The theory of sleep centers connects the dream to the periodic change of the activity of the subcortical centers of sleep and wakefulness, in particular, it found that the biorhythm given by the suprachiasmatic nucleus of the hypothalamus [13]. However, this natural biorhythm is not imperative factors inevitably induces sleep.

III. Cortical sleep theory that sleep comes as a result of irradiation in the cortex and subcortical structures of the inhibitory process of the brain. [7] Sleep may be the reason for the restriction of entering the brain of a new, significant information or action monotonous repetitive stimuli. Information deprivation [14], especially pronounced in people who have lost sight and hearing, leads to constant drowsiness. However, in the study of the visual, parietal association cortex, lateral geniculate nucleus, hippocampus, hypothalamus, thalamus, reticular formation, it was found that the activity of neurons in a variety of structures in the brain during sleep increases considerably $[15,16]$.

IV. Corticosubcortical sleep theory points to the participation and interaction of the cortex and subcortical nuclei in the formation of a dream [17].

V. Visceral sleep theory associates the development of sleep with sensory flows from excitations of the gastrointestinal tract, causing the activation of neurons in the somatosensory cortex [15].

VI. Anabolic concept considers wakefulness as fatigue and sleep as a regenerative process, aimed the completion of energy substances in the brain and in the body as a whole.

VII. Information dream concept implies that the sleep function is the processing of information received in the waking state, the stabilization of the emotional status and participation in the mechanisms of memory.

Wakefulness and sleep are determined by many interrelated factors. None of the modern theory of sleep is not able to explain the biological significance and mechanisms of the relationship of sleep and wakefulness. There is the theory that considers the role of mental activity of the brain in the mechanisms of sleep and wakefulness.

\section{The Phases of Sleep}

Sleep is not a uniform, while sleeping humans and animals exhibit different phase of slow and fast sleep [18-23]. Night sleep a person consists of 4-5 cycles of "slow" sleep and "rapid" sleep. The duration of a complete sleep cycle is relatively constant and in a healthy person is 90-100 minutes. REM sleep occupies $80-75 \%$ of the duration and REM sleep $20-25 \%$. Phase REM sleep is characterized by the deep sleep, accompanied by a lack of consciousness, decreased respiratory rate, heart rate, blood pressure and body temperature, muscle relaxation and decrease movement. This remains an open question of principle that is primary in the initiation phase of slow wave sleep: the mental activity of the brain, or, on the contrary, the neurophysiological mechanisms of REM sleep, deep sleep and cause shutdown of consciousness?

In the dream phase of REM sleep gives way to periods of "rapid eye movement", in which there is low-amplitude EEG activity at a frequency of 30-40 oscillations per second. REM sleep is characterized by the appearance of rapid eye movement, each lasting $0.5-1.5 \mathrm{sec}$., The presence of mental activity of the brain in the form of dreams. Prolonged sleep deprivation and leads to mental disorders. Selective deprivation "rapid" sleep causes emotional irritability, while deepening this state may appear hallucinations, paranoid ideas. This remains an open question what comes first: the neurophysiological mechanisms of REM sleep, shaping dreams, or mental activity of the brain with dreams, triggers REM sleep?

Thus, in the phase of rapid and slow wave sleep there are different manifestations of mental activity of the brain, the role of mental activity of the brain in the formation of the sleep phases and their biological significance remain outside the field of view of researchers.

\section{The structures of the brain involved in the organization of waking and sleeping states}

The active state of the brain during wakefulness provided by the reticular formation. The activation of the reticular formation runs the cortical mechanisms of wakefulness and the inclusion of consciousness [17,24-28]. With the destruction of the reticular formation of the brain stem or transection at the level of the midbrain there is a dream. The blockade of the ascending activating influences on subcortical cerebral cortex occurs deep sleep. By reducing the flow of sensory information reduced ascending activating influence of the reticular formation on the cerebral cortex, and there sleep off with consciousness. Somnogennymi brain structures are involved in the formation of typical sleep behavior and phase synchronized and desynchronized activity of the brain [18,21,22,27].

REM sleep is associated with the activity of synchronizing the EEG brain structures caudal brainstem. Low-frequency stimulation in the solitary tract nucleus is synchronized EEG 
[27]. Backfilling accompanied by increased activity of the neurons in this area. In the experiments of W Hess, T Tokizane shows that the stimulation of the anterior hypothalamus appear on EEG sleep spindles and slow wave activity that is caused by the interaction of the hypothalamus from the midbrain and thalamus structures $[14,24,29]$. The main structure is somnogennoy thalamo-cortical system, which influence the synchronization. WP Koella [30] considers the thalamus of the brain leading synchronizing apparatus [30]. When electrical stimulation of the lateral preoptic area and there is a sleep EEG synchronization [31]. Destruction of this area causes insomnia, cachexia and death of the animals.

According to the neurophysiological studies "slow" sleep starts joint cores. Local destruction of the nuclei leads to chronic insomnia, which can end in death [23]. Of particular significance in the timing mechanism belongs to the orbital cortex. Removing it is accompanied by the disappearance of the characteristic of sleep slow wave activity. Thus, in many areas of brain stimulation can be obtained at timing effects and manifestations of sleep EEG. Structures formed REM sleep, are the upper sections of the reticular nucleus caudal pons and middle sections of oral reticular nucleus of the pons [28,32-34]. Local destruction of these areas entail the disappearance of REM sleep without a material impact on REM sleep and wakefulness.

In the study of the visual, parietal association cortex, lateral geniculate nucleus, hippocampus, hypothalamus, thalamus, reticular formation, it was found that the activity of neurons in a variety of structures in the brain during sleep increases considerably $[15,23]$. All evidence points to the active nature of the neurophysiological processes in sleep and wakefulness, the lack of sleep during the "diffuse inhibition", covering different brain structures. Study of the neurophysiological processes does not allow to understand the nature of mental activity of the brain during wakefulness and sleep.

System interaction neurophysiological and mental activity of the brain

The theory of functional systems, developed PK Anokhin and is widely represented in the work of many other researchers, "paves the conceptual bridge" between the mental and neurophysiological activity of the brain and central nodal points to mechanisms which are associated with the formation of purposeful behavior and origin of emotional and cognitive processes [17]. In the functional System component reflected only the neurophysiological processes of the brain and mental activity is not represented by the brain, which remains a kind of "behind the scenes", only means that it exists.

There is a huge gap between the knowledge of the neurophysiology of the brain and the ideas of his mental functions $[2-4,6,8,17]$. Many prominent scientists have pointed to no attention to this fundamental issue, and the takeaway from the scope of its research activities $[10,11]$. This optimistic view about the possibility of mental activity of the brain cognition expressed IP Pavlov (1951), R.W. Of Sperry (1952), PKAnohin (1968), NP Spondylitis (1990), K.V.Sudakov (2010), $[4,7,9,11,17]$. Modern neurophysiological methods, based on the laws of physics and chemistry, discovered in inanimate nature, do not in themselves make it possible to reveal the nature of the mental functions of the brain.

In all neurophysiological studies, we see only the "outer, tip of the iceberg, the interior, the underwater part" characterizing the mental activity of the brain, remain outside the field of view of researchers, as though it does not exist [35-37]. This explains the "dip" between the understanding of mental and neurophysiological phenomena in the brain, which indicates T. Nagel [6]. In vivo and in particular in the brain may occur such phenomena and physical processes which in principle is not, and can not be inorganic nature. The origins of mental activity are the fundamental properties of the living brain, which is a special form of matter, which has its own physical laws and the specific field.

In our research, we have proposed a fundamentally new approach to the study of the nature of mental activity of the brain. In this case we start from a methodological principle formulated by us, that "mental processes can directly register and study only with the help and participation of the living structures" [35-39]. For the first time demonstrated the existence of "psychogenic field", reflecting the mental activity of the human brain. It presents a paradigm that considers the origin of mental activity and the possibility of the existence of unique to the living brain of the physical phenomena and fields. A schematic diagram of the formation of the subjective state of the brain based on the reverse influence of psychogenic field on neural molecular processes [36,37].

System organization neurophysiological and mental activity of the brain (Figure 1) has two interconnected and united in a single whole subsystem: neurophysiological and psychological [36,37]. Structural and functional neurophysiological components of the system can not carry out their productive activities without the participation of the psychic sphere, as well as mental activity is based on the neurophysiological processes. Neurophysiological layer is the foundation for the perception of the environment and the internal state of the body; for the implementation of various forms of behavior and the regulation of body waste.

At the neurophysiological level, there is the perception of sensory excitation flows from the senses, generated biological motivation, memory components related to storage and storage of information, efferent, team programs, controlling movement, behavior and autonomic reactions occur reflex reactions, automated behavioral acts, due to previously existing prelaunch integration, there is evaluation of results. On the mental 
level, there is judgment of all incoming information the brain, forming social motivation is initiated extract the necessary information from the memory, there is the goal, and there are all the psychological symptoms, such as consciousness, thinking, emotions, etc. Main psychiatric brain functions. Free Will, goal setting, choice of behavior, thought, imagined, and the result of evaluation of achievement occur on a subjective conscious level.

Memory has two components: a neurophysiological and subjective. storing process is carried out on the neurophysiological level, which occurs with the involvement of the subjective activity of the brain. The process of storing all the information in the memory occurs at the neurophysiological level without subjective perception. Recollection and retrieval of information from memory is always going on with the participation of the subjective sphere of mental activity of the brain. Sleep plays a special role in the long-term memory fixation. Traces of perception and memorization of information during wakefulness manifested in the mental activity of the brain in the form of dreams (Figure 1).

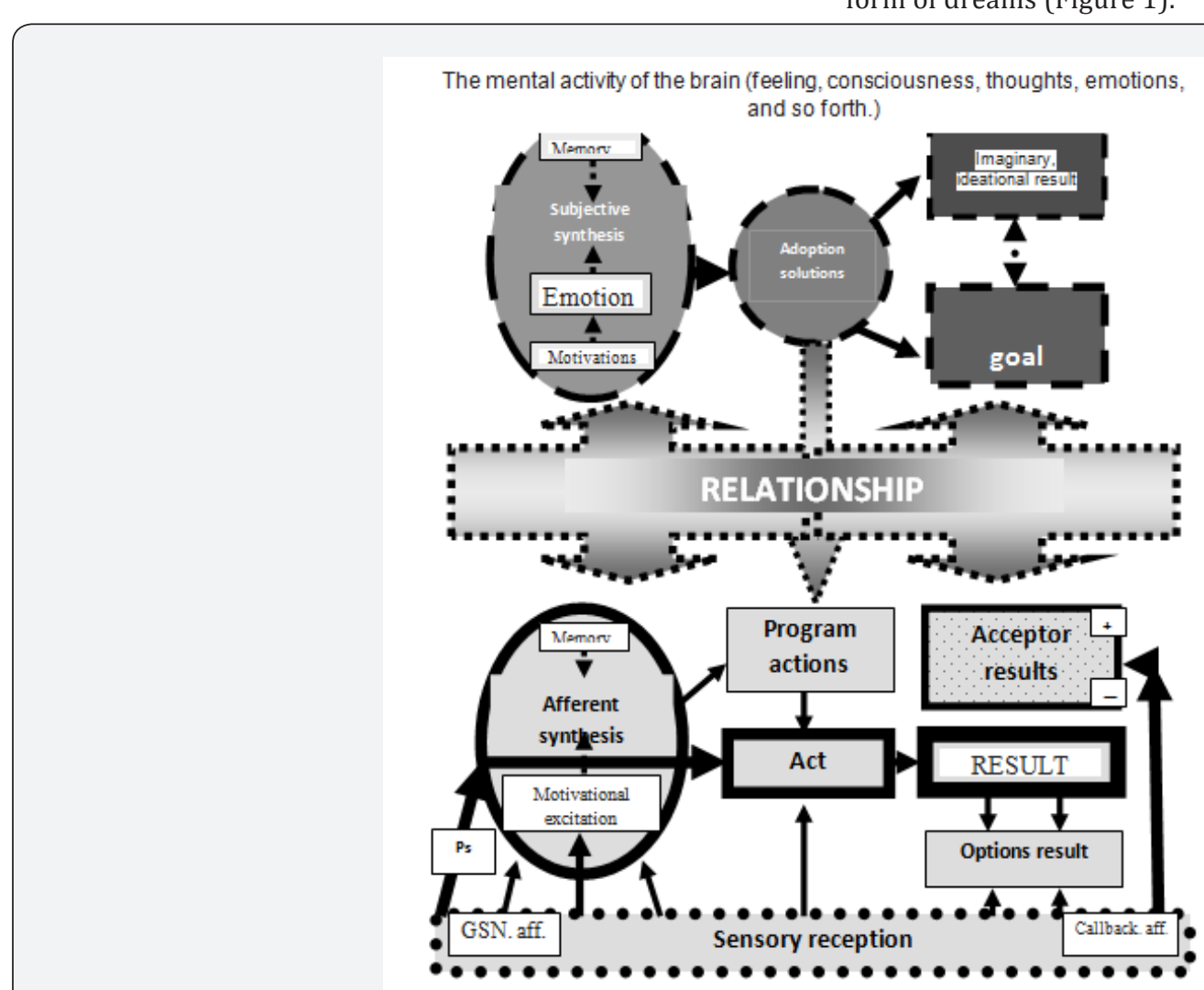

Neurophysiological brain activity levels.

Figure 1: Scheme of the system organization neurophysiological (by PK Anokhin) and mental activity of the brain during wakefulness. Ps: Start-up incentive, OBS. AFF: Situational afferentation, Arr. AFF: Reverse afferent impulses.

In fact, all currently limited memory neurophysiological study mechanisms. Without the involvement of the psychic sphere is impossible to reveal all the mechanisms of memory. Emotions mobilize the body to achieve the objective. Negative emotion impels to action, which would achieve the desired result. Positive emotion creates the effect of satisfaction and reinforcement succeeds behavioral act. Behavior is always focused on the general vector from the negative to the positive emotions. emotions emerged in mental health are converted at the neurophysiological level in emotional reactions. Mental and neurophysiological activity of the brain are interconnected, and two-way communication between them.

\section{System organization of the cycle "sleep-wake"}

Sleep-wake cycle is determined by a complex of factors: circadian biorhythm, cyclic change of sleep and wakefulness, emotional and motivational state, sensory information, fatigue $[13,18-23]$. There is a significant need for biologically awake and motivation, as expressed in the pursuit of an active life. During waking consciousness reflects both the outer world and the inner state of a person. Waking supported sensory information flows from the external and internal environment of the body, activation of brain structures, the presence of the expressed motivations and unmet emotional needs, circadian biorhythm. During wakefulness made mental activity of the brain, including the mind, feelings, emotions (Figure 1).

Falling asleep and sleep are determined by the biological need for sleep. The development of carotid motivation arises from the activation of the neurophysiological processes in the brain in the combined action of a number of factors: the change of the circadian rhythm of the suprachiasmatic 
nucleus of the hypothalamus, the daily stereotype of the day and night, satisfaction of biological and social needs, fatigue, sensory deprivation, decreased motor activity. Driving system organization neurophysiological and mental activity of the brain during sleep is shown in (Figure 2). Motivation sleep is formed on the neurophysiological level, which then manifests itself in the sphere of the subjective mental feeling of drowsiness, lethargy, fatigue, tendency to sleep, and in severe cases, "falling asleep on the go." Attraction to sleep there in the mental health of brain activity at the complex participation of different neurophysiological mechanisms.

If you have any other motivational factors, such as motivating to perform responsible work, the implementation of the carotid motivation can be suppressed volitional consciousness. At the same time, expressed negative emotions and emotional stress interfere with sleep. Emotional stress, initially formed in the mental sphere as a conflict between desired and actual causes insomnia. The relationship between the strong-willed effort, emotional stress and sleep is primarily carried out in the mental activity of the brain. From brain psychiatric desire to sleep is sent to the neurophysiological mechanisms for the formation of sleep and their role in ensuring effective wakefulness (Figure 2) behavioral and autonomic responses, defining the transition to sleep.

If you have a dream disappears consciousness communicates with the external environment of the organism, and together purposeful behavior stops with him. During sleep, there is a continuous interaction of neurophysiological and psychological spheres of activity, which is expressed by dreams, autonomic and motor responses, changes of phases of sleep. In the dream, a person experiences a subjective feeling. This is different from the dream of unconsciousness caused by anesthesia, coma, during which it is impossible awakening. Lack of consciousness during sleep deprives a person of communication with the surrounding reality and, therefore, derived from the psychic sphere of activity of the brain, dreams are fragmentary, emotional, environmental non-deterministic environment directly. However, emotionally charged dreams occur during REM sleep, are related to the mental activity of the brain during wakefulness. Undoubtedly, there is a link with sleep phases of mental activity of the brain. This remains unexplored part of mental activity of the brain in the formation of each sleep phase and their role in ensuring effective wakefulness.

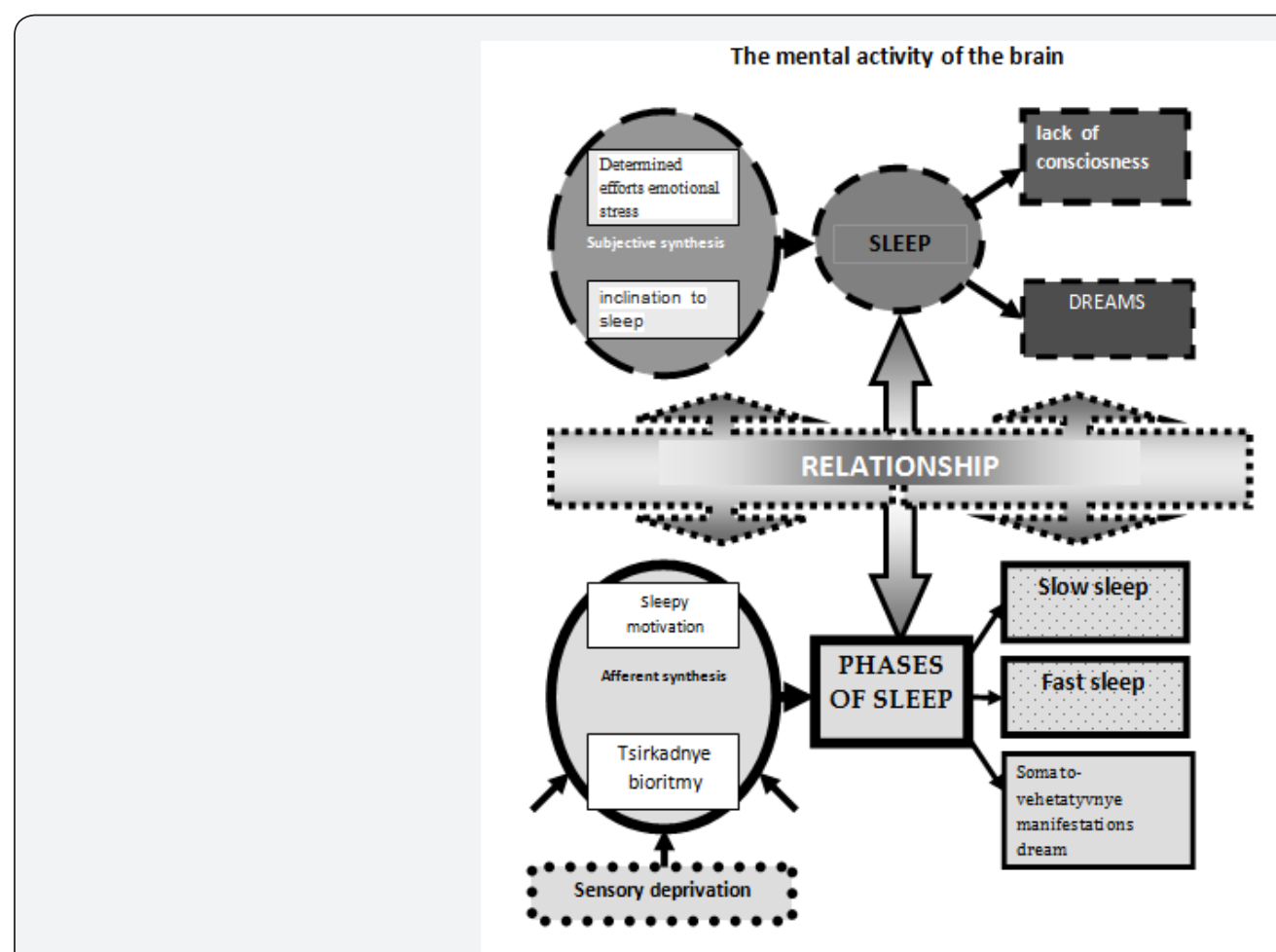

Neurophysiological brain activity

Figure 2: Driving system organization neurophysiological and mental activity of the brain during sleep.

Shutdown of consciousness during sleep is temporary and neurophysiological activation scope of any significant stimulus to mental health problems triggered by consciousness, ie, awakening. The more pronounced carotid motivation, the need a large force of awakening stimulus for the emergence of consciousness, the greater the emotional dissatisfaction manifested by the interrupted sleep. Constant violent awakening can cause emotional stress and neurotic disorders. Waking up after a full sleep enough due to appreciation of the need for sleep and elimination of carotid motivation. As a result, a full sleep is formed motivational excitation natural awakening, leading to wakefulness. Awakening is a positive reinforcement, having a 
positive emotional, which manifests itself in the field of mental activity of the brain. Complete physiological sleep is an antistress factor.

When violent awakening and sleep deprivation occurs a negative emotional state characterized by lethargy, fatigue, headache, depression, drowsiness. Violation of the quality of sleep, insomnia is also accompanied by a marked negative subjective states. Casual emotional stress leads to insomnia, which in turn aggravates the development of stress. The mechanisms of sleep and wakefulness are combined into a coherent system organization, including neurophysiological and psychological processes. During wakefulness formed the motivation that causes sleep, and vice versa, during sleep is the formation of motivation of wakefulness. There is a relationship: waking is a dream, and the dream produces wakefulness. In the wake-sleep cycle, the most important role belongs to the mental activity of the brain. Brain activity during sleep and wakefulness can not be opened without understanding the nature of the mental activity of the brain. The origins of sleep disorders are mental activity of the brain.

\section{Conclusion}

All of the modern idea of sleep and wakefulness are based only on the study of neurophysiological processes, without taking into account the mental activity of the brain mechanisms that are still poorly understood. Unfortunately, neuroscience has developed a skeptical attitude to the scientific study of the nature of the mental activity of the brain, primarily caused by the lack of up to date objective methodology. The mental activity of the brain was beyond scientific research, and for it neurophysiology, whatever exists. Undoubtedly, without the knowledge of the origin of mental activity ideas about the brain are extremely limited, and very far from the truth.

In our research, we have proposed a fundamentally new approach to the study of the nature of mental activity of the brain, based on the methodological principle of "mental processes can directly register and study only with the help and participation of living structures," and introduced the concept and paradigm of systemic organization of mental activity of the brain. Disclose the nature of brain activity can only theory that unifies neurophysiological and mental activity of the brain. To paraphrase a famous saying of M. Jouvet: "Who knows the secret of sleep, the brain learns the secret", it can be said: "Who knows the mental activity of the brain, he knows the secrets of the brain, including the nature of sleep."

\section{Acknowledgment}

This work was supported by the Russian Humanitarian Science Foundation (project No. 16-06-00810,

This work was supported by grant number 16-06-00810 $\mathrm{RFH}$ on "Psychophysiological research of the correlation of emotional stress and the structure of the natural nighttime sleep among students with varying academic performance."
Yumatov Evgeny Antonovich - MD, professor, academician of the International Academy of Sciences,

Ch. n. from. First MGMU them. THEM. Sechenov, Ch. from. Institute of Normal Physiology PKAnohin.

\section{References}

1. Anokhin PK (1969) Psychic form of reflection of reality. Collection of Lenin's theory of reflection and the present. Pavlova Sofia 1(3): 109.

2. Bechterew NP (1974) neurophysiological aspects of human mental activity. Medicine L $151 \mathrm{pp}$.

3. NP spondylitis, Budza PV (1977) Gogolitsyn YL Brain codes of mental activity. The science L 165 C.

4. NP spondylitis Per aspera ... Life (1990) The science of the human brain. The science L 145 pp.

5. Ivanitskii AM (1999) The main mystery of nature: how subjective experiences arise from the processes of the brain. Psychological Journal 20(3): 93-104.

6. Nagel T (2001) conceivable impossible and the problem of mind and body. Problems of Philosophy 8.

7. IP Pavlov (1951) Twenty Years of Objective Study of Higher Nervous Activity (Behavior) of Animals. Full composition of writings. Leningrad Ed. USSR Academy of Sciences.

8. Popper knowledge and psychophysical problem (2008) In defense of the interaction. Trans from English. IV Zhuravlev. Moscow $256 \mathrm{pp}$.

9. Sudakov KV (2010) System mechanisms of mental activity. Neurology and Psychiatry SS Korsakov 110(2): 4-14.

10. Crick F, C Koch (1995) Why neuroscience may be able to explain consiousness. Scientific Amer 273(6): 84-85.

11. Sperry RW (1952) Neurology and the mind-brain problem. Am Sci 40(2): 291-312.

12. Monnier M, Dudler L, Gächter R, Maier PF, Tobler HJ, et al. (1977). The delta sleep inducing peptide (DSIP). Comparative properties of the original and synthetic nonapeptide. Experientia 33(4): 548-552.

13. Klein DC, Moore RY (1991) Reppert S. M. Suprachiasmatic Nucleus: The Mind's Clock. Oxford University Press 467 p.

14. Harrison Y, Horne JA (2000) The impact of sleep deprivation on decision making: a review. J Exp Psychol Appl 6(3): 236-249.

15. IN Pigarev (2013) Visceral sleep theory. Journal of Higher Nervous Activity them IP Pavlovam 63(1): 86-104.

16. Krueger JM, Obal F (1993) A neuronal group theory of sleep function. J Sleep Res 2(2): 63-69.

17. Anokhin PK (1968) Biology and Neurophysiology of the conditioned reflex. Medicine M 3: 548.

18. Vein AM, Hecht K (1989) Son of man. Physiology and pathology. M Medicine $272 \mathrm{pp}$.

19. Vein AM, Sudakov KV, Levin Ya, Yumatov EA, Kovrov GV, et al. (2001) Stages of sleep after the psycho-emotional effects: Customize. Physiological Journal of them Sechenov 87(3): 289-295.

20. Dorokhov VB (2013) Somnology and security profession. Journal of Higher Nervous Activity them. IP Pavlova 63(1): 33-47.

21. VM Kovalzon (2011) Basics somnology. Physiology and neurochemistry wake-sleep cycle. Bean Knowledge Laboratory .

22. Kleitman N (1963) Sleep and Wakefulness. University of Chicago Press. Chicago. 


\section{Psychology and Behavioral Science International Journal}

23. Mircea M Steriade, Robert W McCarley (2005) Brain Control of Wakefulness and Sleep. Springer Science \& Business Media 728 p.

24. Hess W(1929) The mechanisms of sleep. Amer J Psychol 90(4): 386387.

25. Hernandez-Peon RA (1963) Sleep induced by localized electrical or chemical stimulation of the forebrain. Electroencephalog. Clin. Neurophysiol Suppl 24: 188-198.

26. Jasper H (1949) Diffuse projection system. EEG clin Neurophysiol 1(4): 405-419.

27. Moruzzi G (1972) The sleep-waking cycle. Ergeb Physiol 64: 1-164.

28. Rossi GF (1963) Sleep-inducing mechanims in the brain stem. Electroencephalogr Clin Neurophysiol 24S: 113-132.

29. Tokizane T (1965) Hypothalamic control of cortical activity and some observations during different states of sleep. Jouvet Paris Centre Nat Rech Sci p. $151-184$.

30. Koella WP (1984) The organization and regulation of sleep. A review of the experimental evidence and a novel integrated model of the organizing and regulating apparatus. Experientia 40(4): 309-338

31. Clemente CD, Sterman MB, Wyrwicka W (1963) Forebrain inhibitory mechanisms conditioning of basal forebrain induced E.E.G. synchronization and sleep. Exptl Neurol 7: 404-417.
32. Jouvet M (1962) Sur l'existence d'un systeme hypnique ponto-limbique. Ses rapports avec l'activite onirique. In Physiologie de l'hipocampe. Passouant Paris Centre Natl Rech Sci p. 297-330.

33. Jouvet M (1967) Neurophysiology of the states of sleep. Physiol Rev 47(2): 117-177.

34. Jouvet M (1988) The regulation of paradoxical sleep by the hypothalamo-hypophysis. Arch ital biol 126(4): 259-274

35. EA Yumatov (2013) The mental activity of the brain - the «key» to the knowledge. Journal of the International Academy of Sciences. Russian section 1: 35-45.

36. EA Yumatov (2014) The methodology of the study of consciousness in modern psychophysiology. In the book «150 years» Reflexes of the Brain Iintell M 152

37. Yumatov EA (2014) To knowledge of the origin of the brain mental activity. World Journal of Neuroscience 4(2): 170-182.

38. Yumatov EA, Bikova EV, Dzhafarov RN (2013) Long-Distance Effects of Human Subjective Status on the Blood Physicochemical Characteristics. Bull of Experim Biol and Med 155(4): 527-530.

39. Yumatov EA, Bykova EV, Potapova OV, Ragimov AA, Salimov EL (2015) Remote-Field Manifestations of Mental Activity of the Human Brain. World Journal of Neuroscience 5(2): 108-114.

Your next submission with Juniper Publishers will reach you the below assets

- Quality Editorial service

- Swift Peer Review

- Reprints availability

- E-prints Service

- Manuscript Podcast for convenient understanding

- Global attainment for your research

- Manuscript accessibility in different formats ( Pdf, E-pub, Full Text, Audio)

- Unceasing customer service

Track the below URL for one-step submission https://juniperpublishers.com/online-submission.php 